Journal of the Electrochemical Society, 1992, Volume 139, Issue 8, Pages 2196-2201.

ISSN: 0013-4651

DOI: $10.1149 / 1.2221202$

http://www.electrochem.org/

http://scitation.aip.org/getpdf/servlet/GetPDFServlet?filetype=pdf\&id=JESOAN000139000008002196000001\&idty pe $=$ cvips\&prog $=$ normal

(C) The Electrochemical Society, Inc. 1992. All rights reserved. Except as provided under U.S. copyright law, this work may not be reproduced, resold, distributed, or modified without the express permission of The Electrochemical Society (ECS). The archival version of this work was published in Journal of the Electrochemical Society, 1992, Volume 139, Issue 8, Pages 2196-2201.

\title{
Pit Growth in NiFe Thin Films
}

\author{
G. S. Frankel,* J. O. Dukovic,* V. Brusic,* B. M. Rush,**a and C. V. Jahnes \\ IBM Research Division, T.J. Watson Research Center, Yorktown Heights, New York. \\ * Electrochemical Society Active Member. ** Electrochemical Society Student Member. ${ }^{\text {a }}$ Present address: \\ Department of Chemical Engineering, University of California, Berkeley, Berkeley, CA.
}

\begin{abstract}
Pit growth was studied in $80 \mathrm{Ni}-20 \mathrm{Fe}$ sputtered thin films by analysis of images of the growing pits. The pit current density was found to increase with pit growth potential until reaching a limiting value. The limiting current density increased with decreasing film thickness. The mass-transfer resistance to the active pit wall exceeds by an order of magnitude that predicted from a simple radial-diffusion model. It is suggested that the undercut, remnant passive film collapses over the pit wall causing a constriction. A voltage component calculation matches the data rather well and indicates that pit growth below the limiting current density is limited by a combination of ohmic, concentration, and surface activation considerations.
\end{abstract}

The study of pitting in thin films is of interest because of differences in behavior compared to pitting in bulk alloys. Thin films often have different properties and certainly have more stringent requirements in terms of allowable material loss. Furthermore, thin films provide a unique opportunity for studying pit growth since the whole pit is visible during the growth process. As a result, no assumptions need be made regarding the active pit area during growth.

Pits were previously shown to penetrate thin metallic films quickly and reach the substrate $^{1}$. They grow subsequently in a two-dimensional fashion with walls perpendicular to the substrate. This initial study was performed on approximately $1500 \AA$ thick Al films, and the average pit current density was calculated from images of the growing pits. The pit current densities were found to be large (tens of $\mathrm{A} / \mathrm{cm}^{2}$ ) and independent of time during pit growth. There was, however, an influence of pit growth potential. At the highest growth potentials, the pit current density was rather independent of potential, and the pits were very round in shape. At lower potentials, the pit current density varied approximately linearly with potential, and the pits were more irregular in shape. At the lowest growth potentials, the pit perimeters were extremely convoluted, and the calculated pit current density was again independent of potential, although the latter was determined to be an artifact of the calculation as discussed below. Pit growth was described to be under mass-transport control in the highest potential region and under mixed ohmic/charge-transfer control at lower potentials.

The alloy studied in this work is Permalloy, a NiFe alloy. The early investigations of this alloy focused on its oxidation and atmospheric corrosion behavior ${ }^{2-6}$. Recently, studies of the 
aqueous corrosion and pitting behavior of Permalloy have been reported ${ }^{7,8}$. The purpose of the present study is to extend the technique of pit growth measurements in thin films to this different alloy system and to further the understanding of the rate-controlling processes during pitting in thin films.

\section{Experimental Method and Analysis of Data}

The experimental method was similar to that described previously ${ }^{1}$. A brief description indicating differences from the previous study will follow. The samples were 80 atom percent (a/o) Ni-20 a/o Fe films deposited onto quartz substrates by RF diode sputtering from an alloy target in a high-purity argon plasma. Since the samples were very susceptible to crevice corrosion, they were masked with black wax to expose circular areas approximately $0.3 \mathrm{~cm}^{2}$ in size. Samples were then attached to the bottom of a Plexiglas cell by pressing the masked area against a Viton o-ring.

The electrolyte, $0.1 M \mathrm{NaCl}$ made from reagent-grade chemicals and deionized water, was deaerated before and during the experiment with Ar gas bubbling. All potentials were measured against a saturated calomel electrode (SCE). Pit growth was studied under potentiostatic conditions. Several minutes after introduction of the electrolyte, the sample was typically polarized directly to the pit growth potential. For lower growth potentials, pits were first initiated by stepping for about $5 \mathrm{~s}$ to a high potential such as $700 \mathrm{mV}$. The potential was then stepped down to the growth potential.

These NiFe films often generated several pits during one experiment in contrast to the $\mathrm{Al}$ films studied previously which usually developed single pits ${ }^{1}$. As a result, it was not possible to distinguish the current associated with each pit.

The cell cover had a quartz sleeve allowing for insertion of a microprobe for optical viewing of the surface. Images of growing pits were recorded on an optical disk recorder at a rate of one image per second. Some of the images (approximately 1 per $10 \mathrm{~s}$ of growth) were analyzed after each experiment using an image-analysis software package to determine pit perimeter, $P$, and pit bottom area, $A_{\mathrm{b}}$, as functions of time, $t . A_{\mathrm{b}}$ is the area of the quartz substrate exposed by the pit growth. The magnification of the images was such that upon digitization each pixel had an area of $5.4 \mu \mathrm{m} \times 6.7 \mu \mathrm{m}$. As observed in the case of $\mathrm{Al}^{1} P$ was found to increase approximately linearly with $t$, and $A_{\mathrm{b}}$ was found to increase approximately with $t^{2}$. If these two functionalities are assumed, the calculated current density will be constant with time as was discussed previously ${ }^{1}$. In order to avoid forcing this situation, however, the data were fitted in a less constraining fashion using a nonlinear regression fit to the following equations

$$
\begin{gathered}
P=a_{1}\left(t-t_{0}\right)+b_{1}\left(t-t_{0}\right)^{2} \\
A_{\mathrm{b}}=a_{2}\left(t-t_{0}\right)^{2}+b_{2}\left(t-t_{0}\right)^{3}
\end{gathered}
$$

The value of $t_{0}$ reflects the pit initiation time relative to an arbitrary value displayed on the video monitor by a time generator. It was determined in the fit of the data for $P$, which was a threeparameter fit for $t_{0}, a_{1}$ and $b_{1}$. This value of $t_{0}$ was then assumed to be identical in the fit for $A_{\mathrm{b}}$, which became a two-parameter fit for $a_{2}$ and $b_{2}$. This type of fitting typically provided a good description of the experimental data. As described previously ${ }^{1}$, the average anodic pit current density, $\vec{l}_{\mathrm{a}}$, may be calculated from 


$$
\bar{i}_{\mathrm{a}}(t)=\frac{\rho n F}{M} \frac{\partial A_{\mathrm{b}}(t)}{\partial t} \frac{1}{P(t)}
$$

where $\rho, n, F$, and $M$, have the usual meanings. Since differences in dissolution rate may exist at different locations around the pit, $\vec{l}_{\mathrm{a}}$ is a spatial average over the whole active area of the pit. If $P$ and $A_{\mathrm{b}}$ are fitted to the forms shown in Eq. 1 and 2 , the calculated $\vec{l}_{\mathrm{a}}$ will not necessarily be independent of time. This calculated value of $\vec{l}_{\mathrm{a}}(t)$ was then averaged over the growth time of the pit to generate $i_{\mathfrak{c}}^{*}$ The standard deviation for the time average was typically less than $5 \%$, and $\vec{l}_{\mathrm{a}}(t)$ increased slightly with time in some experiments and decreased slightly in others. This indicates that the pit current density was almost constant with time during pit growth and that $i_{\mathrm{a}}^{*}$ is a good representation of

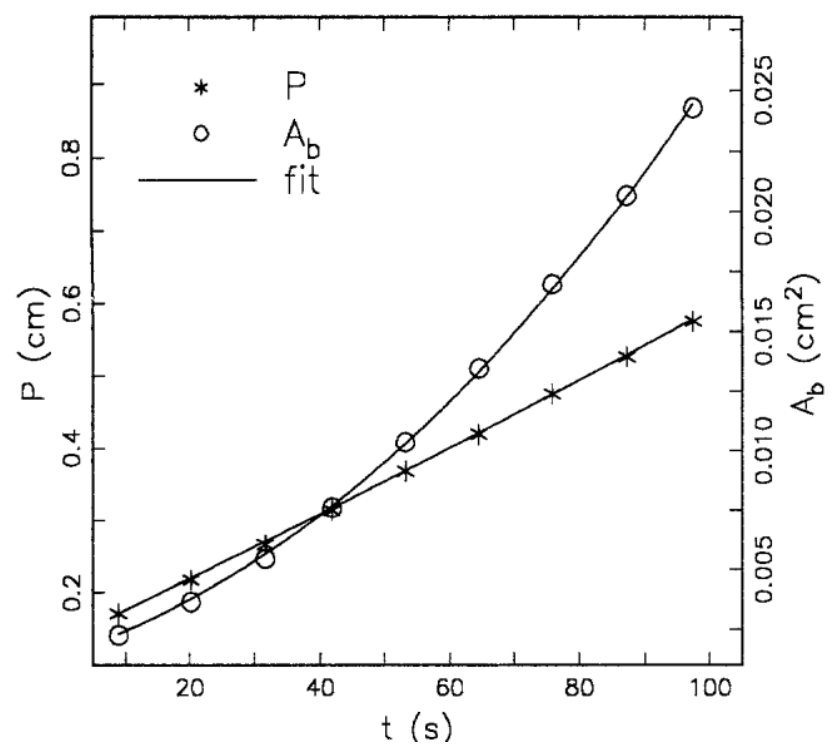

Fig. 1. Pit perimeter and pit bottom area for a pit grown in a $2050 \AA ̊$ thick NiFe film at $700 \mathrm{mV}$ SCE.

$\vec{i}_{\mathrm{a}}(t)$. The time and spatially averaged value of pit current density, $i_{a}^{*}$ was determined as a function of pit growth potential and metal-film thickness. Values of for $n$ and $\rho / M$ were taken to be weighted averages of the pure metal values or 2 equiv/mol and $0.15 \mathrm{~mol} / \mathrm{cm}^{3}$, respectively. Since it was typically not possible to distinguish the current due to each pit, the net current density described previously ${ }^{1}$ (the measured pit current divided by $\bar{x} P(t)$, the active pit area) could not be determined. No gas evolution was observed during growth, however, so the net current density should be identical to the anodic current density calculated from the images of the growing pits. The net current density was calculated for one experiment in which only one pit formed and was found to be very close to the calculated value of $i_{\mathfrak{c}}^{*}$ This indicates that the values used for $n$ and $\rho / M$ are accurate.

\section{Results and Discussion}

Experiments. - Shown in Fig. 1 are values of $P$ and $A_{\mathrm{b}}$ for a typical pit in a NiFe thin film. The solid curves are the fits to Eq. 1 and 2. Figure 2 shows the average anodic current density for the data of Fig. 1 calculated from Eq. 3 using the fitted values for the constants. The 
calculated $\vec{l}_{\mathrm{a}}(t)$ is seen to be almost constant with time, supporting the notion that $i_{a}^{*}$ is a good

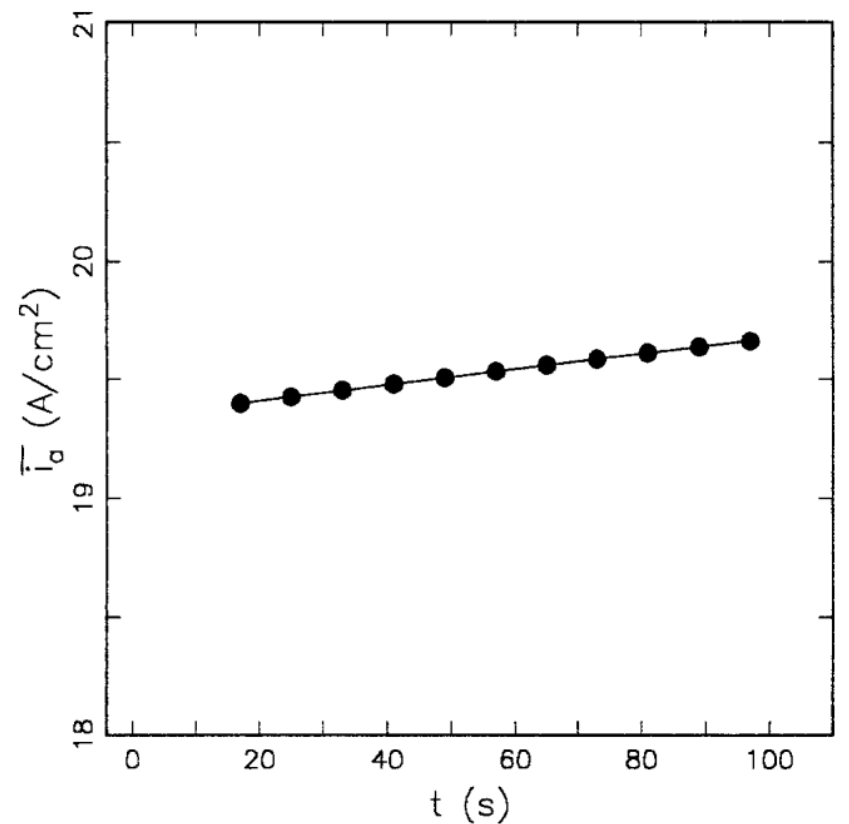

Fig. 2. Current density as a function of time calculated from fit of data in Fig. 1.

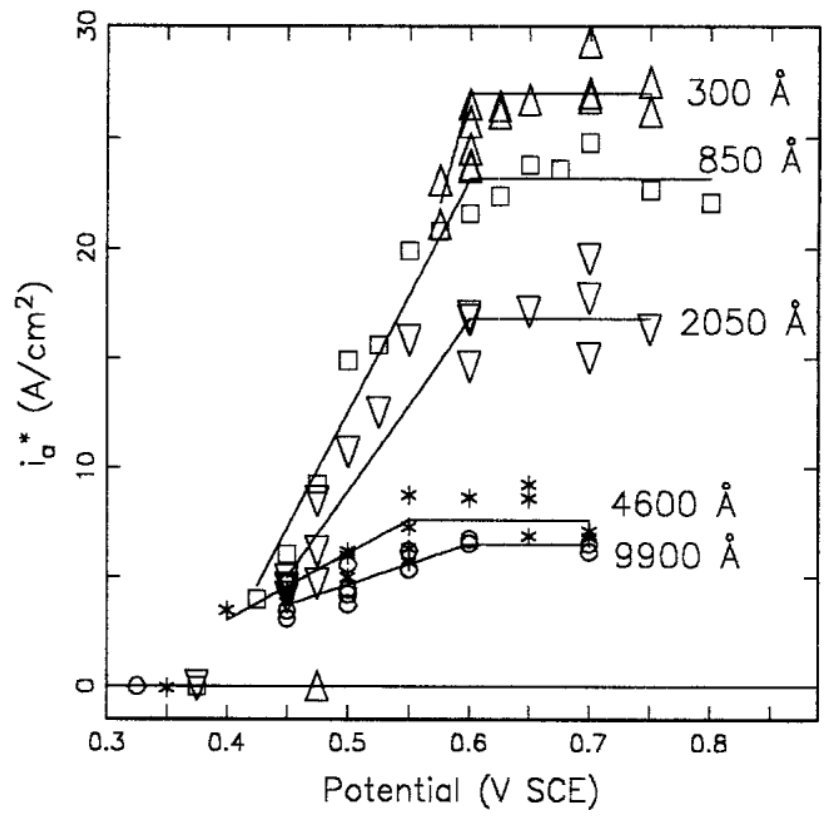

Fig. 3. Average anodic current density as a function of growth potential and film thickness for NiFe films in $0.1 M \mathrm{NaCl}$. Symbols along the potential axis indicate repassivation potentials.

representation of the average current during pit growth. This invariance of pit current density with time was also found for pits in Al thin films and seems to be a characteristic of pits in thin films grown potentiostatically. In thin films there is no increasing dimension to result in an 
increasing diffusion path or ohmic potential drop that would lead to a decrease in pit current density with time, which is commonly observed for large pits in bulk metal samples.

Values of $i_{a}^{*}$ are shown in Fig. 3 as a function of growth potential and metal film thickness for NiFe films from 300 to $9900 \AA$. The data have a form similar to that found for the case of Al thin films: a limiting potential-independent current, $i_{\mathrm{L}}$, at high potentials and a potential-dependent region at lower potentials. No evidence of another potential-independent region at very low potentials was observed. This is likely related to the morphology of the pits which, while similar to the case of $\mathrm{Al}^{1}$, was somewhat different. Outlines of two pits determined by the image analysis program are given in Fig. 4. These pits were in $2050 \mathrm{~A}$ thick NiFe films and were grown at potentials of 700 and $500 \mathrm{mV} \mathrm{SCE}$, respectively. The images were taken at
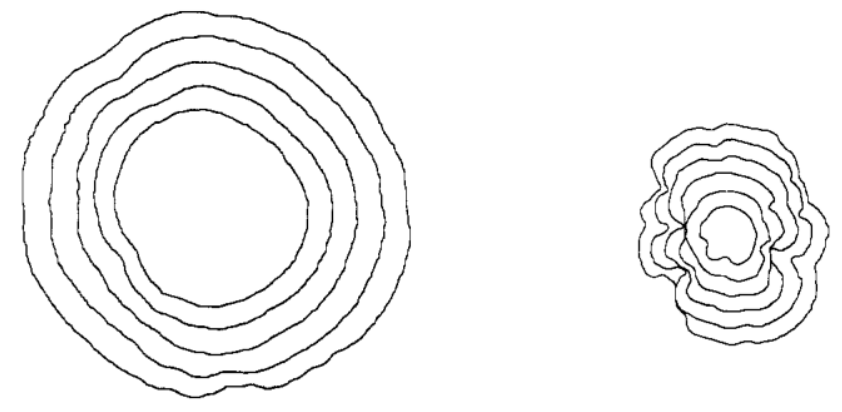

Fig. 4. Perimeters of pits grown in $2050 \AA ̊$ films at (a, left) $700 \mathrm{mV}$ and (b, right) $500 \mathrm{mV}$.

10-s intervals, and it is clear that the higher-potential pit was rounder and grew more rapidly. The lower-potential pit had some regions that grew more slowly than the average, and the perimeter was not very round, but it was not extremely convoluted either. In general, the low-potential pits in the NiFe alloy were less convoluted than pits in $\mathrm{Al}$, where the limited resolution of the image digitization in comparison to the very small features of the perimeters led to underestimates of the pit active area and a potential-independent region at the lowest potentials.

Using similar reasoning to the case of $\mathrm{Al}^{1}$ the round morphology and potentialindependent current density at high potentials are indicative of mass-transfer-controlled growth. The limiting current density, $i_{\mathrm{L}}$, increased with decreasing film thickness. This effect is related to the rate of radially enhanced diffusion from small areas compared to larger ones. More on masstransport effects will be discussed below.

Below $i_{\mathrm{L}}$, the current density varied almost linearly with pit-growth potential. The slope of the line increased with decreasing film thickness. In the previous study ${ }^{1}$, this region was assumed to be under ohmic or mixed ohmic/charge-transfer control. In order to examine the role of metal-film thickness and to assess the rate-determining processes better, a simple model was developed as will presently be described.

Diffusion from pit in a thin film. - Calculations of diffusion from a pit in a thin film were made. To avoid the complexity of solving the transport equations in more than one dimension, 
the system geometry was idealized as follows. First, the curvature of the pit wall was neglected (since the pit diameter greatly exceeds the film thickness during most of the pit's lifetime), allowing the pit wall to be idealized as the vertical face of a semi-infinite straight step, Fig. 5a. This structure is essentially two-dimensional. Second, this structure was idealized as an active semi-cylinder protruding from an inactive plane, Fig. 5b. The radius of the semi-cylinder, $r_{0}$, is such that surface area of the semi-cylinder matches that of the step face. Third, it was assumed that a stagnant diffusion boundary layer extends between the active semi-cylinder and a concentric semi-cylinder of larger radius, $r_{\mathrm{B}}$, with complete mixing outside of this boundary layer. The result is a one-dimensional diffusion problem in cylindrical coordinates.

Since the diffusion resistance from a step profile is not expected to equal that from an equal-area semi-cylinder, the following simple correction was made. Using a commercial finiteelement package (FIDAP, by Fluid Dynamics International), the finite-element method was used to solve for the diffusive flux (Fick's law) in two different two-dimensional configurations. In

a)

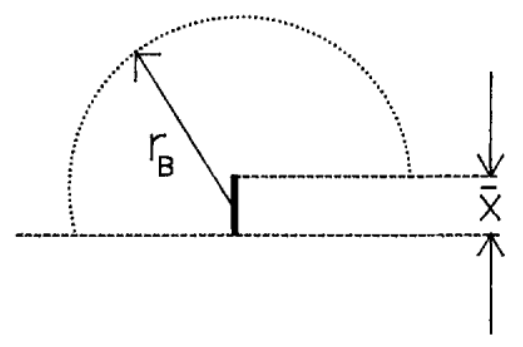

b)

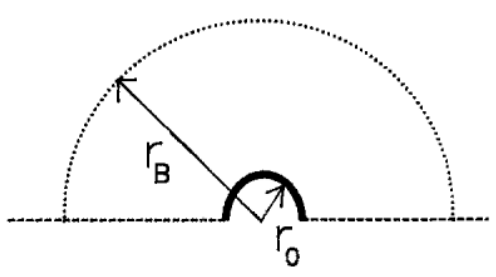

Fig. 5. Schematic representations of pit in thin film: (a) vertical step, (b) semi-cylinder. Bold lines indicate active area.

both cases, the domain was a square box, $20 \times 20 \mu \mathrm{m}$. One entire wall was held at concentration $\mathrm{c}_{1}$ and, except for a small feature at the center of the opposite wall, no flux was allowed at any other boundaries. At the feature surface the concentration $c_{1}$ was imposed. In one case the feature was a $0.1 \mu \mathrm{m}$ vertical step, and in the other case the feature was a protruding semicircle of radius $0.1 / \pi \mu \mathrm{m}$. (The arc-length of both features was $0.1 \mu \mathrm{m}$.) The diffusive flux calculated for a semicircle was 2.0 times higher than that for the step profile. This ratio was adopted as an approximate correction factor in estimating diffusion resistances for step-profiles from simpler calculations on the cylindrical model. Similar factors have been commonly used to account for the complex geometry associated with pits ${ }^{9}$.

The following further simplifying assumptions were also made: $(i)$ consider the alloy to be a pure metal $(\mathrm{Ni})$, (ii) consider the solution to be a simple binary electrolyte $\left(\mathrm{Ni}^{2+}\right.$ and $\left.\mathrm{CI}^{-}\right)$, (iii) assume the boundary-layer radius $r_{\mathrm{B}}=20 \mu \mathrm{m}$, and (iv) ignore hydrolysis and multiple $\mathrm{Ni}$ species in solution. A concentration-dependent diffusion coefficient for the $\mathrm{NiCl}_{2}$ binary electrolyte, $D(c)$, was taken from Stokes et al. ${ }^{10}$ but was modified by the factor of 2.0 to account 
for the higher transport resistance from the real step geometry compared to the idealized semicylindrical geometry. The assumption that the NiFe alloy can be represented by a pure metal is not unreasonable since the solubilities and diffusivities of $\mathrm{Ni}^{2+}$ and $\mathrm{Fe}^{2+}$ are similar.

As a result of the assumptions, the following equation for steady-state diffusion in cylindrical coordinates was solved with the accompanying boundary conditions

$$
\begin{gathered}
\frac{d}{d r}\left(r D(c) \frac{d c}{d r}\right)=0 \\
\frac{d c}{d r}=\frac{-i}{2 F D} \text { at } r=r_{0} \\
c=c_{\mathrm{B}} \quad \text { at } r \geq r_{\mathrm{B}}
\end{gathered}
$$

where $c$ represents the $\mathrm{Ni}^{2+}$ concentration and $c_{\mathrm{B}}$, the bulk concentration, was assumed to be $10^{-}$ ${ }^{9} M$.

The results of this calculation are given in Fig. 6 in terms of the nickel-ion concentration at the pit surface, $c_{\mathrm{s}}$, as a function of film thickness and current density. As expected, the calculations indicate that the current density required to reach a given concentration at the wall of a pit increases as the film thickness decreases. However, further examination reveals a serious problem with these calculations. The vertical lines on each curve denote the experimentally determined mass-transport-limiting current densities for each thickness. The surface $\mathrm{NiCl}_{2}$ concentrations predicted by the model at the observed limiting current densities are far below the known saturation concentration, $c_{\text {sat }}$, of $4.55 M^{11}$. No variation of $D$ or the diffusion-layer thickness within reasonable limits can account for this discrepancy.

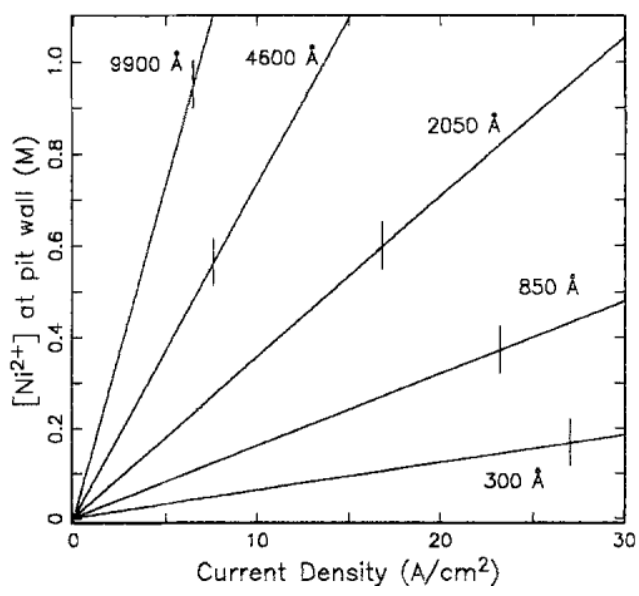

Fig. 6. Surface $\mathrm{Ni}^{2+}$ concentration as a function of current density and film thickness calculated from simple radial model. 


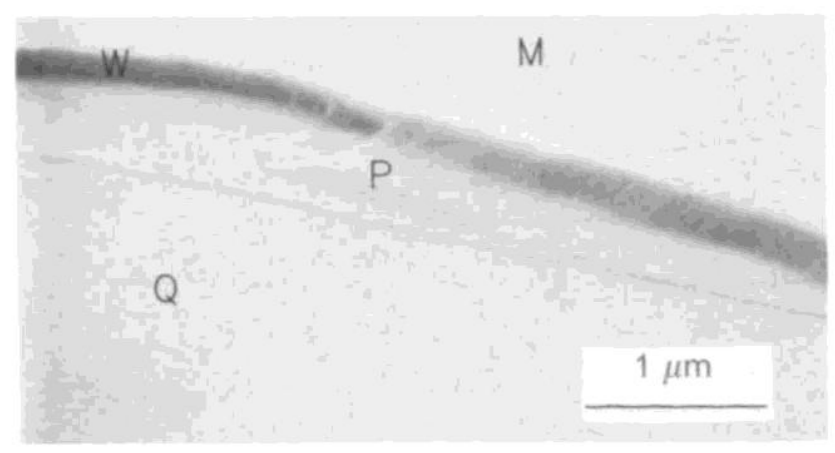

Fig. 7. Scanning electron micrograph of pit wall, $M$, metal film; $Q$, quartz substrate; $P$, undetermined passive film collapsed onto pit bottom; $\mathrm{W}$, pit wall.

This suggests that there exists some additional obstruction to mass transport other than the diffusion boundary layer. In order to obtain 5 to 25 times higher concentration at the surface for a given current density, something must be blocking mass transport.

One characteristic of pits in thin films not shared by pits in bulk alloys is the proximity of the total active surface to the passive film. In the previous study on $\mathrm{Al}^{1}$, it was shown that large pieces of under-eaten passive film remained following pit growth at high potentials. At lower potentials, smaller pieces were pinned down to the many islands that remained within the pits. For Al, however, copious hydrogen gas evolution at the pit wall broke and lifted the passive film. During pit growth at high potentials, in fact, it was sometimes possible to observe with the microprobe large pieces of passive film with attached $\mathrm{H}_{2}$ bubbles extending up into the solution. Evidence for the presence of a remnant passive film for the NiFe alloy is given by scanningelectron-microscopic (SEM) examination of the pit walls, Fig. 7. A piece of the under-eaten passive film is seen in Fig. 7 to be resting on the pit bottom next to the pit wall. It has folded back onto itself and the pit wall on the right side of the micrograph. The samples were prepared for SEM analysis with as little perturbation of the surface as possible by gently washing with water and air drying. However, the position of the remnant passive film during pit growth is likely to be much different from that shown in Fig. 7. Nonetheless, the presence of this remnant passive film after washing and drying indicates that it was in fact present during the pit growth. As mentioned above, however, no gas bubbles were seen for these NiFe films. There was, therefore, no force to lift the passive film up into the solution, and the presence of this film in the diffusion path may have in some way created a barrier to mass transport.

While the mismatch between the measured data and the simple transport model suggests the presence of a constriction (presumed due to the remnant passive film), it says nothing about the geometry of the constriction. The simplest description one can pose for the constriction is a straight passageway of length $L^{*}$ and width $x^{*}$, shown schematically in Fig. 8. This allows a onedimensional model of mass transfer in this region as follows

$$
D \frac{d c}{d y}=-\frac{i_{\text {constric }}}{n F}
$$

where $y$ is the distance coordinate along the constriction as shown in Fig. 8 and $i_{\text {constric }}=i_{\mathrm{a}}^{*}\left(\bar{x} / x^{*}\right)$ is the current density in the constricted region. This passageway is assumed to exist in series with the radial transport zone previously assumed; the details of how the two pathways might be 
connected are ignored for simplicity. The ratio $L^{*} / x^{*}$ can be determined for pits in films of each thickness knowing that $c(y=0)=c_{\mathrm{s}}=c_{\text {sat }}$ when $i_{a}^{*}=i_{\mathrm{L}}$

$$
\frac{L^{*}}{x^{*}}=-\frac{n F}{i_{L} \bar{x}} \int_{c_{\text {sat }}}^{c^{*}} D d c
$$

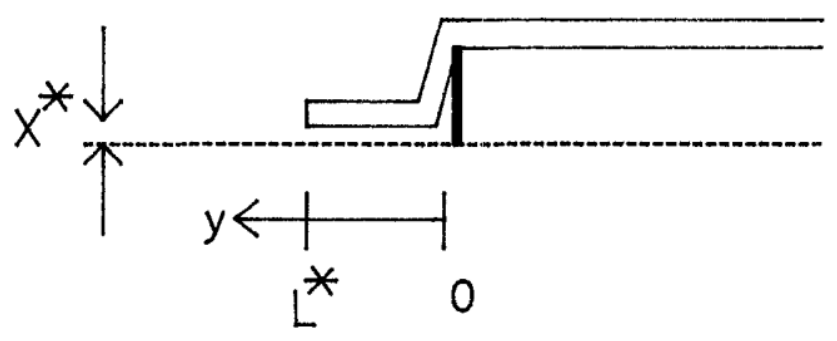

Fig. 8. Schematic representation of pit in thin film as vertical step with remnant-passive-film constriction. Bold line indicates active area.

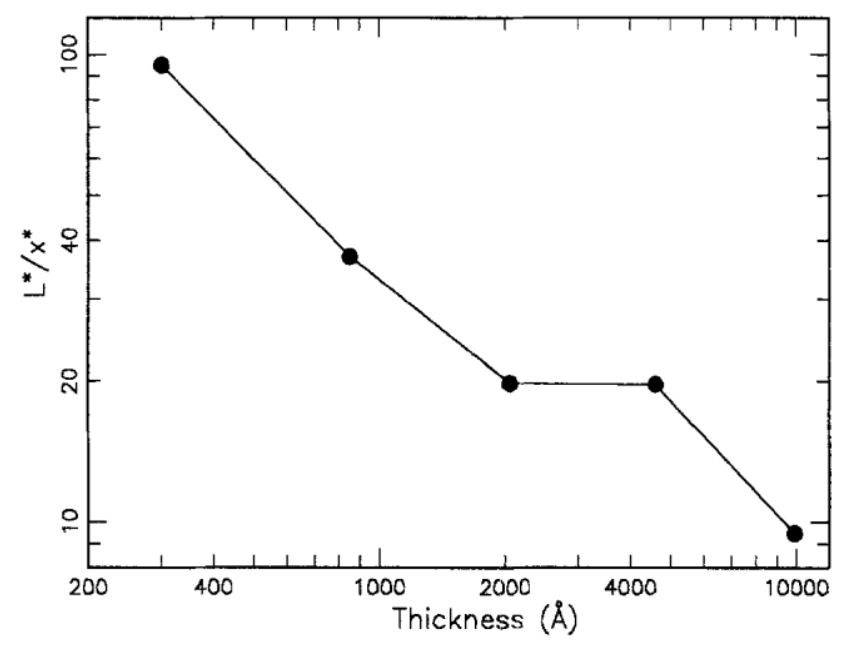

Fig. 9. Geometric factor describing constriction, $L^{*} / x^{*}$, for pits in different film thicknesses.

where the boundary condition $c^{*}$ may be determined from the solution to the cylindrical problem described above. The values for the ratio $L^{*} / x^{*}$ were found to vary from about 10 to 100 depending on metal film thickness as is shown in Fig. 9. This calculation is essentially a fit of the data which provides a constriction of appropriate magnitude to assure that the surface concentration reaches saturation at the limiting current density for each film thickness, Fig. 10. Given the large values of this ratio, it is extremely unlikely that the remnant passive film protrudes directly from the edge of the pit parallel to the pit bottom. It is more likely that the 


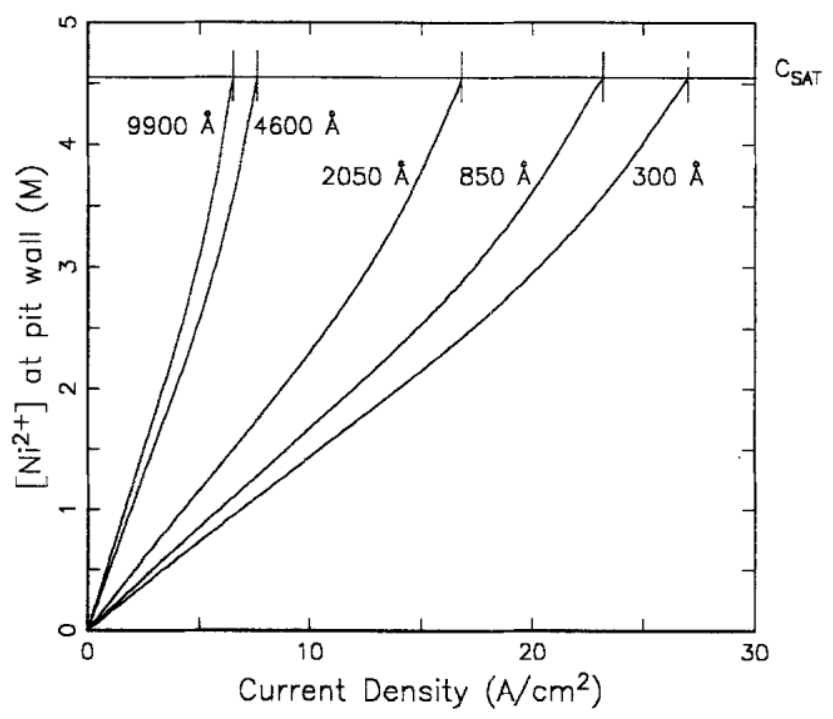

Fig. 10. Surface $\mathrm{Ni}^{2} *$ concentration as a function of current density and film thickness calculated from pit model containing constriction of dimension $L * / x^{*}$ given in Fig. 9.

remnant passive film collapses down onto the pit wall and bottom in a fashion suggested by Fig. 8. Perhaps the constriction ratio is higher for thinner metallic films because $x^{*}$ is smaller. As described above, the current density for a given pit is almost constant with time during the time of pit growth. This indicates that, while the nature of the obstruction is different for pits in films of different thicknesses, the obstruction maintains a steady configuration during pit growth.

Voltage component calculation.-A remnant passive film that impedes mass transfer should also increase the ohmic resistance of the system. In order to examine the influence of such a constriction on the behavior below $i_{\mathrm{L}}$, the slopes of the $i$ - $E$ curves below $i_{\mathrm{L}}$ were compared to slopes predicted from a voltage-component calculation which includes the extra ohmic drop due to the constriction. The various contributions to the total potential difference were calculated using the assumptions stated above but with the addition of a constriction in series with $L^{*} / x^{*}$ as given in Fig. 9. The total potential drop between the reference electrode and the pit wall, $E_{\text {tot }}$, may be written as

$$
E_{\mathrm{tot}}=\eta_{\mathrm{s}}+\eta_{\mathrm{c}}+\Delta V_{\Omega}+E_{\mathrm{T}}
$$

where $\eta_{\mathrm{s}}$ is the surface overpotential, $\eta_{\mathrm{C}}$ is the concentration overpotential, $\Delta V_{\Omega}$ is the ohmic potential drop, and $E_{\mathrm{T}}$ is the thermodynamic difference between the reference electrode and an electrode of the same kind as the active surface located at the position of the reference electrode as described by Newman ${ }^{12}$. The surface overpotential was calculated using Tafel kinetics with a concentration-dependent exchange current density, $i_{0}{ }^{12}$

$$
\begin{aligned}
\eta_{\mathrm{s}}=\frac{R T}{(1-\beta) n F} & \ln \frac{i}{i_{0}(\mathrm{c})} \\
& =\frac{R T}{(1-\beta) n F} \ln \frac{i}{i_{0,1 \mathrm{M}}\left(1000 \times \mathrm{c}_{\mathrm{s}}\right)^{1-\beta}}
\end{aligned}
$$


where the symmetry factor $\beta$ is assumed to be $0.5, c_{\mathrm{S}}$ is the $\mathrm{Ni}^{2+}$ concentration at the surface, and $i_{0.1 \mathrm{M}}$ is the exchange current density at $c_{\mathrm{s}}=1 \mathrm{M}$. The determination of $i_{0.1 \mathrm{M}}$ is discussed below. The concentration overpotential was calculated using the assumption of a simple binary electrolyte, and constant activity using ${ }^{12}$

$$
\eta_{\mathrm{c}}=\frac{-3 R T}{2 F} \int_{\mathrm{c}_{\mathrm{s}}}^{c_{\mathrm{B}}}\left(1-t_{+}\right) d \ln c
$$

where $t_{+}$is the concentration-dependent $\mathrm{Ni}^{2+}$ ion transference number taken from Stokes et al. ${ }^{10}$. The ohmic potential drop was calculated by dividing it into contributions from three regions in series: the constricted path adjacent to the active pit wall, the concentration boundary layer beyond the constriction, and the bulk electrolyte beyond the boundary layer

$$
\begin{gathered}
\Delta V_{\Omega}=\int_{0}^{L^{*}} \frac{i}{\mathrm{\kappa}} d s+\int_{\tau_{0}}^{r_{\mathrm{B}}} \frac{i}{\mathrm{\kappa}} d s+\int_{r_{\mathrm{B}}}^{r_{\infty}} \frac{i}{\mathrm{\kappa}} d s \\
=\int_{0}^{L^{*}} \frac{i_{\mathrm{a}}^{*} \bar{x}}{x^{*} \mathrm{\kappa}} d s+\int_{r_{0}}^{r_{\mathrm{B}}} \frac{i_{\mathrm{a}}^{*} r_{0}}{r_{\mathrm{\kappa}}} d r+\int_{r_{\mathrm{B}}}^{r_{\infty}} \frac{i_{\mathrm{a}}^{*} r_{0}}{r_{\kappa_{\mathrm{B}}}} d r
\end{gathered}
$$

where $s$ represents a distance coordinate along the ohmic pathway, $\mathrm{K}$ is the concentrationdependent conductivity taken from Stokes et al. ${ }^{10}$, and $\mathrm{K}_{\mathrm{B}}=0.01 \Omega^{-1} \mathrm{~cm}^{-1}$ is the bulk conductivity. An example of the various calculated voltage components is given in Fig. 11 for the case of a $850 \AA$ thick film. Also shown in this figure are the data from Fig. 3 for $850 \AA$ thick films. $E_{\mathrm{T}}$ was taken to be $-580 \mathrm{mV}$, a weighted average of the reversible potentials for pure $\mathrm{Ni}$ and Fe as given by Pourbaix ${ }^{13}$ but corrected for the SCE scale. The position along the potential axis (but not the shape) of $\eta$ shifts depending on the value of $i_{0.1 \mathrm{M}}$. The value of $i_{0.1 \mathrm{M}}$ was thus adjusted to overlap the $E_{\text {tot }}$ curve with the data points in the region below $i_{\mathrm{L}}$.

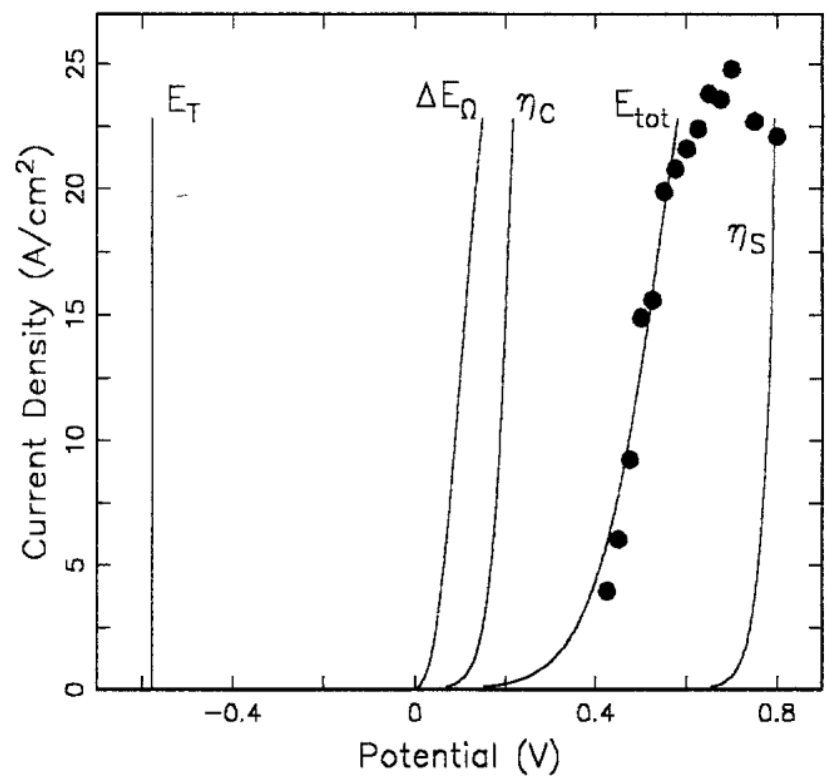

Fig. 11. Calculated voltage components for pit in 850 Á thick film. 
In this fashion, $i_{0.1 \mathrm{M}}$ was determined to be $2.5 \times 10^{-6} \mathrm{~A} / \mathrm{cm}^{2}$ resulting in $i_{0}$ values ranging from 8 $\times 10^{-7}$ to $5.4 \times 10^{-6} \mathrm{~A} / \mathrm{cm}^{2}$ as $c_{\mathrm{s}}$ varied from 0.1 to $4.5 \mathrm{M}$. This formalism clearly displays how the influences of thermodynamics (through the reversible potential, $E_{\mathrm{T}}$ ) and kinetics (through the exchange current density and $\eta_{s}$ ) determine the position of the $E_{\text {tot }}$ curve and thus the relative susceptibility to pitting. However, $E_{\mathrm{T}}$ and $\eta_{\mathrm{s}}$ do not play the largest role in limiting pit growth as the growth potential is increased since they are relatively independent of current density. The components with the lowest slopes in the $i-E$ plot, or the largest $d E / d i$, will have the largest ratelimiting influence.

The slope of each component of the potential, evaluated at a current density equal to half of the limiting current density for each thickness, is given in terms of $d E / d i$ in Fig. 12 along with the fitted slopes of the experimental data. The slope of the calculated total overpotential is seen to match the experimentally determined slope quite well, as is also evident in Fig. 11. The slope of the ohmic potential drop is the largest component for all thicknesses, but significant

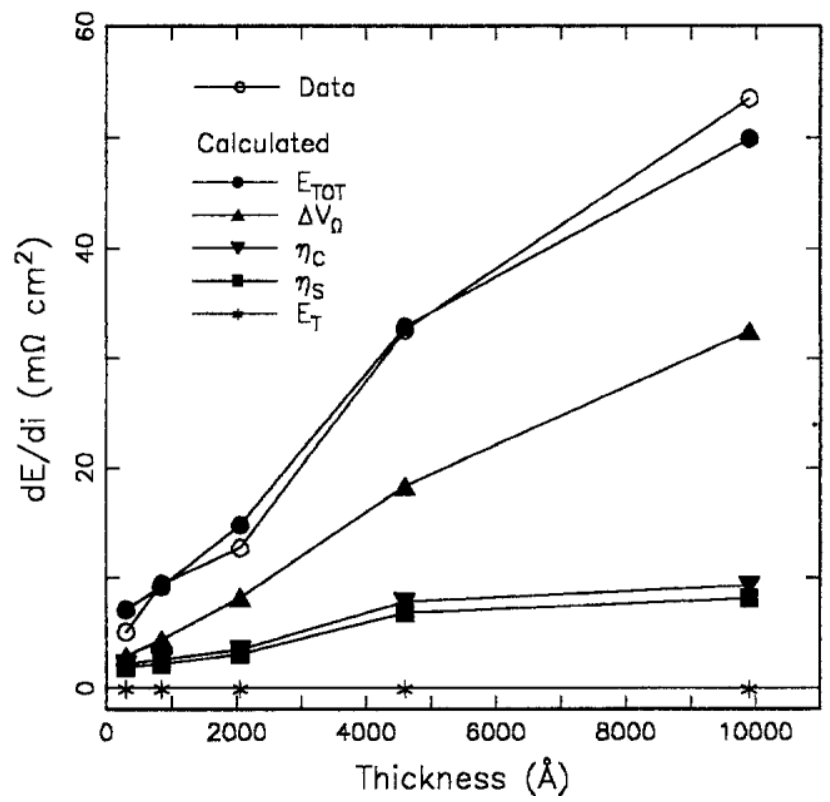

Fig. 12. Slope of $i-E$ curves and calculated voltage components evaluated at half of the limiting current density for different film thicknesses.

contributions are made by the other factors, especially at lower thickness where the slope of the ohmic potential drop is lower. Therefore, the earlier assumption of ohmically controlled pit growth in this region ${ }^{1}$ was not strictly correct since both activation and concentration polarization also play a role.

Since the slopes of the calculated total potentials match the experimental data reasonably well, the hypothesis of a constriction to achieve a surface concentration of $c_{\text {sat }}$ at the observed limiting current density is not in contradiction with the behavior at lower current densities. In fact, the ohmic potential drop associated with the constriction was the largest part of the ohmic potential drop component only for the thinnest samples. The concentration and hence the conductivity were highest in the constriction region, which acted to limit the ohmic drop there.

The calculations allow for the passage of current at potentials as low as the reversible 
potential. This is contradicted by observations of pit repassivation. In Fig. 3, the symbols on the potential axis where $i=0$ indicate repassivation potentials determined by experiments in which the potential was slowly stepped downward after some period of growth and the pit image was recorded. The repassivation potential was taken to be the potential at which the pit stopped growing. Repassivation potentials for pits in thin films are more reproducible than for pits in bulk materials because of the absence of a growing dimension as described above. However, the repassivation potential increases as the film thickness decreases, indicating that it is not a material property. Furthermore, for most of the thicknesses the repassivation potentials are more noble than the potential-axis intercepts of the extrapolated linear regions. While hydrolysis of the cation was not treated in this work, it would result in a $\mathrm{pH}$ reduction approximately proportional to the $\log$ of the cation concentration ${ }^{14}$. Repassivation is likely associated with a critical $\mathrm{pH}$ and thus critical value of $c_{\mathrm{s}}$. As shown above, $c_{\mathrm{s}}$ depends upon both current density and film thickness. The repassivation potential increases with decreasing film thickness because the current density required to maintain the critical concentration increases.

Finally, it should be noted that while others have addressed the chemistry and transport in a pit in a more thorough manner ${ }^{14,15}$, this simple analysis of pits in thin films is useful because the steady-state nature of these pits allows direct comparison of calculations with experiments.

\section{Conclusions}

The anodic current density of pits in NiFe thin films was measured as a function of growth potential and metal film thickness, and the following observations were made:

1. The pit current density was constant with time and increased with pit-growth potential until reaching a limiting value that depended on film thickness.

2. In order for saturation to occur at the pit surface for current densities equal to the observed values, a constriction in the diffusion path must exist. It is suggested that the undereaten remnant passive film collapses over the pit wall during growth.

3. A calculation of the various potential components resulted in a good match to the experimental data in terms of the slope $d E / d i$.

IBM T. J. Watson Research Center assisted in meeting the publication costs of this article.

\section{REFERENCES}

1. G. S. Frankel, Corros. Sci., 30, 1203 (1990).

2. W.-Y. Lee, G. Scherer, and C. R. Guarnieri, This Journal, 126,1533 (1979).

3. W.-Y. Lee, J. Eldridge, and C. R. Guarnieri, ibid., 124, 1747 (1977).

4. D. W. Rice, J. C. Suits, D. Nepela, and T. Tremoureux, J. Appl. Phys., 50, 7089 (1979).

5. D. W. Rice and J. C. Suits, ibid., 50, 5899 (1979).

6. D. W. Rice, J. C. Suits, and S. J. Lewis, ibid., 47, 1158 (1976).

7. C. H. Lee, D. A. Stevenson, L. C. Lee, R. D. Bunch, R. G. Walmsley, M. D. Juanitas, E. Murdock, and J. E. Opfer, in Corrosion of Electronic and Magnetic Materials, P. Peterson, Editor, ASTM, Philadelphia, PA (1991).

8. J. G. Bornstein, C. H. Lee, L. A. Capuano, and D. A. Stevenson, J. Appl. Phys., 65, 2090 (1989).

9. K. J. Vetter and H. H. Strehblow, in Localized Corrosion NACE-3, R. W. Staehle, B. F. Brown, J. Kruger, and A. Agrawal, Editors, NACE, Houston, TX (1974). 
10. R. H. Stokes, S. Phang, and R. Mills, J. Sol. Chem., 8, 489 (1979).

11. F. Hunkeler and H. Bohni, in Corrosion Chemistry in Pits, Crevices, and Cracks, A. Turnbull, Editors, HMSO, London (1987).

12. J. S. Newman, Electrochemical Systems, Prentice Hall, Englewood Cliffs, NJ (1991).

13. M. Pourbaix, Atlas of Electrochemical Equilibria in Aqueous Solutions, NACE, Houston, TX (1974).

14. J. R. Galvele, This Journal, 123, 464 (1976).

15. J. N. Harb and R. C. Alkire, ibid., 138, 2594 (1991). 